\title{
FitClipse: A Tool for Executable Acceptance Test Driven Development
}

\author{
Shahedul Huq Khandkar, Shelly Park, Yaser Ghanam, and Frank Maurer \\ Department of Computer Science, University of Calgary \\ 2500 University Dr. NW, Calgary, AB \\ Canada T2N $1 \mathrm{~N} 4$ \\ \{s.h.khandkar, sshpark, yghanam, fmaurer\} @ucalgary.ca
}

\begin{abstract}
FitClipse is an Eclipse plug-in for facilitating Executable Acceptance Test Driven Development. The tool allows the users to edit acceptance tests, automatically generate fixtures, execute tests and represent the test results graphically including an option to view the test results history. The tool helps with regression testing because it can distinguish between requirements tasks that were never tackled before and tasks that were already completed but are now failing again. FitClipse currently supports GreenPepper and Fit framework.
\end{abstract}

Keywords: Executable Acceptance Test Driven Development, Testing Framework, Fit, Fitnesse, Greenpepper.

\section{Introduction}

Extreme Programming ensures the quality of a software development project through unit testing and acceptance testing. Unit tests are used to verify the functionality of the software system from the developer's perspective and acceptance tests are used to verify whether the functionalities of the system meet the requirement of the customer. The agile methods suggest that there should be an acceptance test for every user story and a user story should not be considered completed until the software implementation passes all acceptance tests.

Executable Acceptance Test Driven Development (EATDD) is an extension of Test Driven Development (TDD). It is also known as Customer Test-Driven Development. While TDD focuses on ensuring the system design and stability from the developer's point of view using the unit tests, EATDD starts from the customer's perspective to help developers better understand the requirements and validate their development with the customer's requirements.

There are several commercial and open source frameworks that support automated acceptance tests. But due to the lack of proper IDE support, it becomes time consuming and tedious for developers to write and maintain these acceptance tests. FitClipse [1] is an Eclipse plug-in that solves this problem by providing the IDE support for common acceptance test frameworks and additional features to extend the benefits even more.

P. Abrahamsson, M. Marchesi, and F. Maurer (Eds.): XP 2009, LNBIP 31, pp. 259-260, 2009.

(C) Springer-Verlag Berlin Heidelberg 2009 


\section{The Executable Acceptance Test Tool}

FitClipse supports Fit [2] and Greenpepper [3] framework. The earlier version used a wiki based framework FitNesse [4] to store the tests. But many developers expressed that they want to store the tests with the development code to keep track of the changes better. However, FitNesse requires a web server to host the test specifications. To overcome this dependency, the current version of FitClipse replaced FitNesse with its own file system, which allows the code and the tests to be stored together in a version control system.

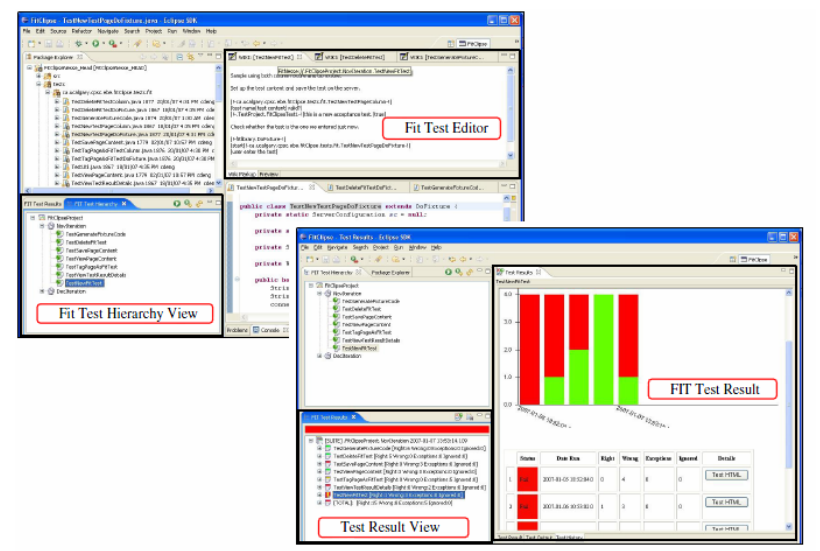

Fitclipse helps the test specification with a built-in WYSIWYG editor, which can help write the test specifications within the IDE more easily. Fitclipse can automatically generate the fixture stubs while you are designing the acceptance test specifications. Fitclipse comes with a test refactoring capability. It can synchronize any later change in the acceptance test (i.e. defining new test scenario, changes in test data) with related fixtures and helps reorganize the tests by automatically identifying the necessary fixture changes. FitClipse provides an analysis on the progress of the software development project using the test results history. The test result contains a statistical report and possible causes of test failures (i.e. exceptions). Often there is considerable delay between defining the acceptance test and its first successful pass. It is important to be able to distinguish between tasks that were never passed before and tasks that were already completed but whose tests are now failing for regression testing purpose. FitClipse is available for download at http://ase.cpsc.ucalgary.ca/ index.php/FitClipse/ FitClipseFrameWorkInstallation.

\section{References}

1. Deng,C., Wilson, P., Maurer, F., FitClipse: A Fit-based Eclipse Plug-in For Executable Acceptance Test Driven Development, Proc. of the XP 2007, Come, Italy 2007, Springer

2. Fit, http:// fit.c2.com

3. Greenpepper, http://www.greenpeppersoftware.com

4. Fitnesse, http://fitnesse.org 